\title{
The Lancet Neurology
}

\begin{tabular}{|c|c|}
\hline Manuscript Number: & THELANCETNEUROLOGY-D-21-00089 \\
\hline Article Type: & Linked Commentary \\
\hline \multicolumn{2}{|l|}{ Keywords: } \\
\hline Corresponding Author: & $\begin{array}{l}\text { Michael P Lunn, MRCP PhD } \\
\text { MRC Centre for Neuromuscular Disease, National Hospital for Neurology and } \\
\text { Neurosurgery and Department of Molecular Neuroscience, UCL Institute of Neurology, } \\
\text { Queen Square, London, UK. } \\
\text { London, UNITED KINGDOM }\end{array}$ \\
\hline First Author: & Michael P Lunn, MRCP PhD \\
\hline Order of Authors: & Michael P Lunn, MRCP PhD \\
\hline Manuscript Region of Origin: & UNITED KINGDOM \\
\hline
\end{tabular}


Preventing expensive harms in the treatment of Guillain-Barré syndrome: more is not better Lunn MP

Centre for Neuromuscular Disease, National Hospital for Neurology and Neurosurgery, Queen Square, London WC1N 3BG UK

02034488121

Guillain-Barré syndrome (GBS) is one of the best known, and possibly best understood, inflammatory neurological diseases. Despite this, it has arguably made less therapeutic progress in 30 years than we would like. The paper by Walgaard et al. \{reference $\}$ in this edition of Lancet Neurology clarifies the lack of effect of second doses of IVIG in the treatment of GBS, highlights real potential harms with second doses and should sweep away some of the inertia associated with the search for new treatments.

GBS is a post-infectious, macrophage-associated inflammatory polyradiculoneuropathy, that in many cases is humorally targeted and complement-mediated. GBS is the commonest cause of acute neuromuscular paralysis in the western world (1). At presentation, patients are manifesting the effects of acute immune attack targeted at peripheral nerve epitopes. Removing the soluble mediators responsible is therapeutically effective and from the mid-1980s plasma exchange (Plex) became the initial treatment. The definitive trial (2) conclusively demonstrated the effect was neither to cure GBS, nor prevent progression or improve endpoint disability, but to halve the time to improve a grade on the GBS Disability Scale, reduce the time to walk unaided by more than a month and to halve the time to ventilator independence in severe cases. Treatment later than four weeks had no benefit.

In 1988 Kleyweg at al. (3) published their positive (and the first) experience of the use of intravenous immunoglobulin (IVIG) in 6 of 8 patients with GBS. IVIG had already found use in other immune diseases; why should it not work in GBS? Kleyweg described two GBS patients who improved with IVIG following Plex, and one of the remaining four 'IVIG-responsive' patients improved whilst receiving a second IVIG dose 21 days after the first; the idea of the 'second IVIG dose' was born. IVIG has never been compared to placebo in adults, but its effect has equivalence to Plex (4). In this respect, a standard $2 \mathrm{~g} / \mathrm{kg} \mathrm{IVIG} \mathrm{dose} \mathrm{essentially} \mathrm{'helps} \mathrm{the} \mathrm{patient} \mathrm{get} \mathrm{better} \mathrm{more} \mathrm{quickly'} \mathrm{(there} \mathrm{is} \mathrm{a}$ small meta-analytical effect of Plex on at one year). Second doses were further encouraged by observational studies of 'treatment related fluctuations' (5) and where a lesser incremental rise in IgG post IVIG was associated with poor outcome (6) but these were observational data with innate bias. We also now recognise some GBS as 'acute chronic inflammatory demyelinating polyradiculoneuropathy' (a-CIDP) (7) which may respond to repeated IVIG as CIDP does.

Walgaard performed a placebo controlled, randomised trial (RCT) of the second dose of IVIG in GBS with a poor presentation prognosis to answer a question that has remained since Kleyweg et al. (3): Does a second dose of IVIG given a short time after the first dose improve the outcome of patients with a poor presenting prognosis? Those of us treating GBS for many years have all given some second IVIG doses when we are watching helplessly, apparently doing nothing, for the return of limb movement in our severely affected GBS patients. A large observational cohort within the Inflammatory GBS Outcomes Study suggested no effect of a second IVIG dose (8). But Walgaard's RCT definitively answers the question of whether a second dose has any effect? And that answer is a 
conclusive 'No' for any outcome measure.. More importantly, second doses are associated with temporally related harms of significant thromboembolic complications. And these harms are expensive; Walgaard has not estimated the cost of care for the treatment of complications. IVIG has a finite supply and inappropriate use reduces the supply of IVIG for other indications; and in financial terms if $25 \%$ of IVIG treated GBS patients in the UK were given a second dose, the annual NHS cost would be about GBP1.75 million for no measured gain.

IVIG is a highly purified blood product with uses in neurology, haematology, rheumatology and infectious disease. Its many proposed, and evidence supported, modes of action influence innate and acquired immunity (9). Different effects are thought to be more prominent in some diseases than others. However, when a pharmaceutical seems to be a 'jack of all trades', it is also the 'master of none', and we should intensify our search for new and more effective therapy.

Our new approach must be to find treatments that rapidly neutralise the immediate and ongoing damage happening as the patient presents. One of the range of complement inhibitors are an obvious choice and these demand large scale RCTs to demonstrate efficacy on the back of safety shown in small studies $(10,11)$. The emerging class of FcRn inhibitors or IgG metabolising enzymes, such as imlifidase, may also find a niche. In parallel, educational efforts to increase the early recognition of GBS in the Emergency Department will ready clinicians for implementing earlier treatment. Eventually appropriate treatment 'at the door' by increasing awareness of 'Nerve Attack' (analogous to widespread 'Brain Attack' stroke awareness programmes) will hopefully improve outcomes.

In the interim, we need to stop using our precious IVIG resource as 'second doses' in severe GBS and concentrate on supporting our patients through sometimes prolonged recovery without inadvertently adding additional harm.

\section{Sources of Funding: None}

Acknowledgements: MPL is supported by the University College Hospitals London NHS Foundation Trust Biomedical Research Centre

\section{Competing Interests:}

\section{References}

1. Willison HJ, Jacobs BC, van Doorn PA. Guillain-Barre syndrome. Lancet. 2016;388(10045):717-27.

2. Plasmapheresis and acute Guillain-Barre syndrome. The Guillain-Barre syndrome Study Group. Neurology. 1985;35(8):1096-104.

3. Kleyweg RP, van der Meche FG, Meulstee J. Treatment of Guillain-Barre syndrome with highdose gammaglobulin. Neurology. 1988;38(10):1639-41.

4. Hughes RA, Swan AV, van Doorn PA. Intravenous immunoglobulin for Guillain-Barre syndrome. Cochrane Database Syst Rev. 2014(9):CD002063.

5. Kleyweg RP, van der Meche FG. Treatment related fluctuations in Guillain-Barre syndrome after high-dose immunoglobulins or plasma-exchange. J Neurol Neurosurg Psychiatry. 1991;54(11):957-60. 
6. Kuitwaard K, de Gelder J, Tio-Gillen AP, Hop WC, van Gelder T, van Toorenenbergen AW, et al. Pharmacokinetics of intravenous immunoglobulin and outcome in Guillain-Barre syndrome. Ann Neurol. 2009;66(5):597-603.

7. Ruts L, Drenthen J, Jacobs BC, van Doorn PA, Dutch GBSSG. Distinguishing acute-onset CIDP from fluctuating Guillain-Barre syndrome: a prospective study. Neurology. 2010;74(21):1680-6.

8. Verboon C, van den Berg B, Cornblath DR, Venema E, Gorson KC, Lunn MP, et al. Original research: Second IVIg course in Guillain-Barre syndrome with poor prognosis: the non-randomised ISID study. J Neurol Neurosurg Psychiatry. 2020;91(2):113-21.

9. Chaigne B, Mouthon L. Mechanisms of action of intravenous immunoglobulin. Transfus Apher Sci. 2017;56(1):45-9.

10. Davidson AI, Halstead SK, Goodfellow JA, Chavada G, Mallik A, Overell J, et al. Inhibition of complement in Guillain-Barre syndrome: the ICA-GBS study. J Peripher Nerv Syst. 2017;22(1):4-12.

11. Misawa S, Kuwabara S, Sato Y, Yamaguchi N, Nagashima K, Katayama K, et al. Safety and efficacy of eculizumab in Guillain-Barre syndrome: a multicentre, double-blind, randomised phase 2 trial. Lancet Neurol. 2018;17(6):519-29. 